\title{
Factors Affecting the Use of Health Facility for Childbirth in Rural Tanzania: A Literature Review
}

\author{
Fabiola Vincent Moshi* \\ School of Nursing and Public Health, University of Dodoma, Tanzania
}

Submission: May 01, 2018; Published: May 24, 2018

*Corresponding author: Fabiola Vincent Moshi, Assistant Lecturer, School of Nursing and Public Health, University of Dodoma, Tanzania, Email: fabiola.moshi@gmail.com

\begin{abstract}
Background: Forty million births in developing regions were not attended by skilled health personnel, but by traditional birth attendants or a relative in 2012, and over 32 million of those births occurred in rural areas. The aim of this literature review was to ascertain factors which affecting the use of health facility for childbirth in Tanzania. Methods PubMed and Google Scholar databases were used to get published articles on factors which affect the use of health facility for childbirth.

Results: Fourteen factors were identified and grouped under four themes 1) Birth preparedness practice 2) Male involvement 3) Gender roles 4) social economic factors. Challenges in comparing results between studies include differences in methods, context-specificity and the substantial overlap between complex variables.

Conclusion: Previous studies have reported different factors which affect the use of health facilities for childbirth ranges from institutional factors, community factors, family factors and individual factors. High impact intervention studies are needed to work out the cost effective strategies which can be used to improve the use of health facilities for childbirth.

Keywords: Unborn child; Maternal deaths; Survival of neonates; Global under-five deaths; Demographic; Health survey; Birth preparedness; Obstetric, Literate pregnant women; Attending antenatal; Prevailing illiteracy; Inefficient infrastructure; Poor transport system; Neonatal morbidity; Mortality rates; Male partners; Blood donor; Obstetric emergencies
\end{abstract}

\section{Introduction}

Forty million births in developing regions were not attended by skilled health personnel, but by traditional birth attendants or a relative in 2012, and over 32 million of those births occurred in rural area [1].

Home birth with assistant of unskilled birth attendant is risky to both mother and unborn child [2]. It is estimated that 293,300 maternal deaths occurred in 2013 worldwide [3]. Most of these deaths occurred in Sub Saharan Africa (62\%) and South Asia (24\%) which altogether account for $86 \%$ of maternal mortality worldwide [1]. The Tanzanian estimated maternal mortality ratio is 556/100,000 [2].

The survival of neonates depends very much on investment in maternal care, especially access to skilled antenatal care, delivery and early postnatal services [4]. In 2013 more than 2.5 million neonates died within their first 28 days of life worldwide, contributing up to $45 \%$ of global under-five deaths [4].

Health facility births have shown improvement from 2010 demographic and health survey and 2015 demographic and health survey. In 2010, 50\% of births occurred in health facility [5] while in 2015 facility birth increased to 63\% [2]. Despite of the increase we still have $27 \%$ (16\% in urban and $46 \%$ in rural) of women who choose home childbirth over health facility birth. One wonders why those women choose home childbirth with unskilled birth attendants over health facility birth. The aim of the review was to ascertain factors which affect health facility birth in Tanzania.

\section{Methods}

PubMed and Google Scholar databases were used to get published articles on factors which affect the use of health facility for childbirth. Fourteen factors were identified and grouped under four themes 1) Birth preparedness practice 2) Male involvement 3) Gender roles 4) social economic factors.

\section{Findings and Discussion}

In the review of previous studies, the author grouped the factors into four main themes. These are as follows 


\section{Theme 1: Birth preparedness and complication readiness practice}

Studies have reported that the practice of birth preparedness and complication readiness is positively associated with health facility birth $[6,7]$. When a pregnant woman is prepared for health facility child birth, there is an increased chance of using health facility for childbirth. This is probably because when a pregnant women is prepared (has all items needed during childbirth), this particular women gain a courage of using health facility for childbirth. Some pregnant women may opt to have their baby born at home simply because they did not prepare the required birthing items. Birth preparedness and complication readiness are encouraged and emphasized during antenatal visits. The shortcoming of the routine is that no effort is taken to assess if a pregnant woman has prepared the required items needed for childbirth. On top of that little is done to assist the expecting couples on income generating activities which will assist them to be able to prepare for childbirth.

Previous studies have worked out some predictors of birth preparedness and complication readiness; to mention few are:- maternal literacy, availing of antenatal services, parity of a pregnant mother, and knowledge on obstetric danger signs. Literate pregnant women $[8,9]$, attending antenatal [6], being pregnant for the first time [6], being able to mention three or more danger signs [10] were more likely to prepare for childbirth and hence use health facility for childbirth. When these predictor factors are closely analyzed, women from rural community where the burden of illiterate are more likely not able to prepare for childbirth and hence choose home birth under assistance of unskilled birth attendants.

The principle and practice of birth preparedness and complication readiness (BP/CR) in a third world setting where there is prevailing illiteracy, inefficient infrastructure, poor transport system, and unpredictable access to skilled care provider show high potentiality of reducing the existing high maternal and neonatal morbidity and mortality rates.

\section{Theme 2: Role of male partners}

There is a growing awareness and acceptance that men have an important influence on women's health and have distinct reproductive health needs of their own. Reaching out to men as partners may improve spousal communication and may help in early decision-making for seeking care if complications arise [10].

A husband's positive involvement can take many forms, including transporting his wife to a qualified provider, providing household money to make that visit, giving helpful informational support during pregnancy, and offering emotional support during labor and childbirth [11].

When men accompany their wives to hospitals, they have more access to reproductive health information and could result in greater communication between men and women on subjects related to reproductive health and childcare. This improved inter spousal communication could enhance pregnancy planning, birth preparedness and complication readiness [11].

However, men consider maternity units as exclusively meant for women [12]. The attitude of health staff and poor conditions in health centers imply that even husbands who accompany their wives to clinics are often ignored or made to hang around somewhere. This behavior encourage men to give high priority to making plans for naming ceremonies rather than critical components of birth preparedness such as deciding on place of delivery, skilled assistance and identification of a blood donor. The reproductive health facility infrastructures in many developing countries explain it all. From the welcoming from health workers to the setting of clients movement in many health facilities men find it not conducive for them. All these make male feel like they are not responsible in antenatal checkups and childbirth. More worrisome is the lack of plans for decisionmaking and savings for obstetric emergencies [12]

\section{Theme 3: Perception on risks associated with pregnancy}

Personal perceptions of risks is a representation of the individual's culture, including their beliefs, values and upbringing, as well as the influences of the individual's social network, which can include family members, spouses, friends, and community members [13].

Some previous literature has documented that knowledge and awareness of the possibility of medical risks do not avert parents from a home birth decision. Those who chose to give birth at home accepted that there was a risk of complications, but perceived these to be due to fate. Technical risks were considered a consequence of the decision to give birth in hospital, and were perceived to be avoidable [14]. Low risk perception among both pregnant women and their spouses act as a barrier in choosing health facilities for childbirth. Some attitudes like fatalistic attitude where a pregnant woman decide to choose home childbirth by a belief that there is a super natural power that control the birthing process. If the super natural power has decided that you will have safe delivery, you will have it regardless of the place of childbirth [14]. Such attitude makes pregnant women engage in risky behavior.

\section{Theme 4: Influence of gender roles on choosing place of childbirth}

A gender relations analysis reveals power relations between men and women. Women carry the major share of the responsibility for the well-being of the household in most societies. This responsibility is rarely matched by autonomy to make decisions or by access to necessary resources [15].

Research has shown that women with less autonomy have to get permission from husbands, in laws or other family members before seeking health care and in some cultures women need someone to accompany them to a health facility. Those who work for cash are more likely to participate in health care decision- 
making, making major household purchases, daily household purchases and visits to her family or friends than those who are not employed and those who do not work for cash [16].

\section{Theme 5: Social economic factors}

Social economic factors play a substantial role in deciding the place of childbirth. Lack of money [17], distance a woman has to walk to reach health facility $[17,18]$, lack of privacy in health facility facilitate [17] and staff attitude towards delivering women [17] facilitates choice of home childbirth over health facility birth.

\section{Conclusion}

Studies of the factors which affect the use of health facility for childbirth concentrate on improving birth preparedness practice, male involvement, risk perception, gender roles and social economic factors. To come up with valid conclusions, it is important to consider as many influential factors as possible in any analysis of determinants of health facility childbirth.

\section{References}

1. United nation (2014) The Millenium Development Goals Report 2014 [Internet]. United Nations, New York, USA.

2. Ministry of Health (MoH) [Zanzibar], National Bureau of Statistics (NBS), Office of the Chief Government Statistician (OCGS) and I. Tanzania Demographic and Health Survey and Malaria Indicator Survey 2015-2016.

3. Naghavi M, Wang H, Lozano R, Davis A, Liang X, et al. (2015) Global, regional, and national age-sex specific all-cause and cause-specific mortality for 240 causes of death, 1990-2013: A systematic analysis for the Global Burden of Disease Study 2013. Lancet 385(9963): 117-171.

4. UNICEF (2014) Level and Trends in Child Mortality [Internet]. 2014. Maternal, newborn, child and adolescent health, p. 36.

5. National Bureau of Statistics (2010) Tanzania Demographic and Health Survey.

6. Karkee R, Lee AH, Binns CW (2013) Birth preparedness and skilled attendance at birth in nepal: Implications for achieving millennium development goal 5. Midwifery 29(10): 1206-1210.
7. Magoma M, Requejo J, Campbell O, Cousens S, Filippi V, et al. (2010) High ANC coverage and low skilled attendance in a rural Tanzanian district: a case for implementing a birth plan intervention. BMC Pregnancy Childbirth 10: 13.

8. Karkee R, Binns CW, Lee AH (2013) Determinants of facility delivery after implementation of safer mother programme in Nepal: a prospective cohort study. BMC Pregnancy Childbirth 13(1): 193.

9. Urassa DP, Pembe AB, Mganga F (2012) Birth preparedness and complication readiness among women in Mpwapwa district, Tanzania. Tanzan J Health Res 14(1): 42-47.

10. Urassa DP, Pembe AB, Mganga F (2012) Birth preparedness and complication readiness among women in Mpwapwa district, Tanzania. Tanzan J Health Res 14(1): 1-7.

11. Iliyasu Z, Abubakar IS, Galadanci HS, Aliyu MH (2010) Birth preparedness, complication readiness and fathers' participation in maternity care in a northern Nigerian community. Afr J Reprod Health 14(1): 21-32.

12. Nanjala M, Wamalwa D (2012) Determinants of Male Partner Involvement in Promoting Deliveries by Skilled Attendants in Busia, Kenya. Glob J Health Sci 4(2): 60-67.

13. Moshi F, Nyamhanga T (2017) Understanding the preference for homebirth; an exploration of key barriers to facility delivery in rural Tanzania. Reprod Health 14(1): 132.

14. Danforth E, Kruk P, Mbaruku G, Galea S (2009) Household Decisionmaking about Delivery n Health Facilities: Evidence from Tanzania. J Heal Popul Nutr 27(5): 696-703.

15. Bloom SS, Wypij D, Gupta MDAS (2001) Dimensions of Women ' S Autonomy and the Influence on Maternal Health Care Utilization in a North Indian City. Demography 38(1): 67-78.

16. Furuta M, Salway S (2006) Women's position within the household as a determinant of maternal health care use in nepal. Int Fam Plan Perspect 32(1): 17-27.

17. Mrisho M, Schellenberg JA, Mushi AK, Obrist B, Mshinda H, et al. (2007) Factors affecting home delivery in rural Tanzania. Trop Med Int Heal 12(7): 862-872.

18. Mpembeni RN, Killewo JZ, Leshabari MT, Massawe SN, Jahn A, et al. (2007) Use pattern of maternal health services and determinants of skilled care during delivery in Southern Tanzania: implications for achievement of MDG-5 targets. BMC Pregnancy Childbirth 7: 29.

\section{Your next submission with Juniper Publishers will reach you the below assets}

